\title{
Evaluación de la actividad cicatrizante de hierba mosquera (Cronton elegans Kunth) en ratones (Mus musculus)
}

\section{Evaluation of the cicatrising activity of hierba mosquera (Cronton elegans Kunth) in mice (Mus musculus)}

\author{
${ }^{1}$ Cecilia Toaquiza Aguagallo*, ${ }^{2}$ Cristina Tigse Moposita, ${ }^{1}$ Elizabeth Escudero Vilema, ${ }^{1}$ Aída \\ Miranda Barros, ${ }^{3}$ Víctor Guangasig Toapanta
}
${ }^{1}$ Grupo de Investigación de Tecnología y Atención Farmacéutica - Escuela Superior Politécnica de Chimborazo, Riobamba, Ecuador
${ }^{2}$ Hospital de Especialidades Eugenio Espejo. Ministerio de Salud Pública, Quito, Ecuador ${ }_{3}$ Universidad Técnica de Ambato, Ambato, Ecuador
*cecytoaquiza@gmail.com

\begin{abstract}
El objetivo del presente estudio fue evaluar la actividad cicatrizante del extracto de Hierba mosquera (Croton elegans Kunth) en ratones (Mus musculus). Con el extracto etanólico concentrado se realizó cuantificaciones espectrofotométricas para flavonoides totales expresados en porcentaje de rutina y de compuestos fenólicos mediante el micrométodo de Folin-Ciocalteau, y determinación colorimétrica de tanino expresado como ácido tánico. En la evaluación de actividad cicatrizante se utilizaron 18 ratones (Mus musculus) albinos con peso de 35 a 40g, dos meses de edad, 12 machos y 6 hembras provenientes del bioterio de la facultad de Ciencias (ESPOCH). Divididas en 6 grupos experimentales, 3 para cada tratamiento: extracto etanólico de hierba mosquera en concentraciones del 20\%, 40\% y $80 \%$, el control negativo fueron ratones con heridas sin tratamiento, y para controles positivos se aplicaron eterol y crema base de acetato de prednisolona con sulfato de neomicina. Se midió la longitud de la herida y examen histopatológico del corte de piel del lugar de lesión. El contenido total de flavonoides fue $80 \mu \mathrm{g}$, contenido total de fenoles $2.07 \mu \mathrm{g}$ y la concentración de compuestos tánicos $24,36 \mu \mathrm{g}$. El test Tukey con nivel de significancia 0,05\% reveló que el extracto etanólico al 80\% tuvo mayor efectividad. El extracto etanólico al 80\% de hierba mosquera expone reconstitución total de tejido en menor tiempo demostrando actividad cicatrizante.
\end{abstract}

Palabras Claves: Hierba Mosquera (Cronton elegans Kunth), ratones (Mus musculus), actividad cicatrizante, extracto etanólico

The objective of the present study was to evaluate the cicatrizant activity of hierba mosquera extract (Croton elegans Kunth) in mice (Mus musculus). With the concentrated ethanolic extract, spectrophotometric quantifications performed for total flavonoids expressed as a percentage of rutin and phenolic compounds by means of the Folin-Ciocalteau micromethod, and colorimetric determination of tannin expressed as tannic acid. In the evaluation of healing activity, 18 albino mice (Mus musculus) weighing 35 to $40 \mathrm{~g}$, two months old, 12 males and 6 females from the faculty of sciences (ESPOCH). Divided into 6 experimental groups were used, 3 for each treatment: ethanolic extract of hierba mosquera in concentrations of $20 \%, 40 \%$ and $80 \%$, the negative control were mice with wounds without treatment, and for positive controls, eterol and prednisolone acetate base cream were applied with neomycin sulfate. The length of the wound and the histopathological examination of the skin cut at the site of injury measured. The total content of flavonoids was $80 \mu \mathrm{g}$, total phenol content $2.07 \mu \mathrm{g}$ and the concentration of tannic compounds $24.36 \mu \mathrm{g}$. The Tukey test with significance level $0.05 \%$ revealed that the $80 \%$ ethanolic extract was more effective. The $80 \%$ 
ethanolic extract of hierba mosquera exposes total tissue reconstitution in less time, demonstrating cicatrizant activity.

Keywords: Hierba Mosquera (Cronton elegans Kunth), mice (Mus musculus), healing activity, ethanolic extract

Fecha de recepción: 03-10-2018

Fecha de aceptación: 31-01-2019

\section{INTRODUCCIÓN}

La gran diversidad de la flora ecuatoriana ha sido reconocida y estudiada desde hace mucho tiempo, y desde siempre reconocida por ser inmensamente rica en plantas útiles (5172 especies) de las cuales el 60\% son medicinales. Según la enciclopedia de plantas útiles del Ecuador en el país existen 144 familias (17\%) que incluyen 825 especies de plantas que son utilizadas para aliviar heridas y lesiones (1).

Las familias de mayor uso para aliviar heridas y lesiones son Asteraceae, Solanaceae y Euphorbiaceae, numerosas de las especies incluidas favorecen la pronta cicatrización, sobre todo de heridas causadas por objetos corto-punzantes, entre ellas son muy conocidos el matico (Aristeguietia glutinosa), en la Sierra, y la sangre de drago (Croton lechleri), en la Amazonía. La hierba mosquera (Croton elegans kunth) incluida también en esta categoría no reporta estudios previa comprobación científica de esta actividad farmacológica (1).

Según el catálogo de Plantas Vasculares del Ecuador Croton elegans Kunth es una especie endémica del Ecuador perteneciente a la familia Euphorbiaceae (2). De acuerdo a la literatura botánica, esta planta es un arbusto de la vegetación seca interandina, que crece entre 1500 y 3500 msnm (3). Presenta muchas aplicaciones en la medicina tradicional ecuatoriana, se utiliza como antiinflamatorio, en el tratamiento de dolor de muela, heridas amigdalitis y verrugas. También se ha utilizado en el tratamiento de reumatismo, gota, neuralgia y bronquitis (4).

Se atribuye a compuestos fenólicos actividades antiinflamatorias y cicatrizantes; y se ha identificado la presencia de los mismos en hierba mosquera (4). El objetivo de la presente investigación fue evaluar la actividad cicatrizante del extracto de Croton elegans Kunth en ratones (Mus musculus).

\section{MATERIALES Y MÉTODOS}

\section{Material vegetal}

La identificación taxonómica de la hierba mosquera (Croton elegans Kunth) se lo realizó en el Herbario de la Escuela Superior Politécnica de Chimborazo (ESPOCH) y fue recolectada en la parroquia Picaihua de la provincia de Tungurahua a $2600 \mathrm{msnm}$ y temperatura promedio de $15^{\circ} \mathrm{C}$.

\section{Material de laboratorio}

PREPARACIÓN DE LOS EXTRACTOS: Se pesaron para cada extracto $30 \mathrm{~g}$ de material vegetal secado en estufa (Memmert) con circulación forzada de aire a $40{ }^{\circ} \mathrm{C} \pm 1{ }^{\circ} \mathrm{C}$, el material seco se pulverizó hasta un tamaño de partícula máximo de $0.5 \mathrm{~mm}$, y fueron macerados con éter, etanol (40 $\%$ ) en zonicador (Branson 3510) durante 3 horas a temperatura de $25^{\circ} \mathrm{C} \pm 1{ }^{\circ} \mathrm{C}$, luego se filtró y conservó los extractos para los análisis en frascos ámbar a $4^{\circ} \mathrm{C}$ (5) (6).

ENSAYO FITOQUÍMICO: La identificación cualitativa de metabolitos secundarios de los extractos se realizó según tamizaje fitoquímico general (7).

\section{CONTROL DE CALIDAD DEL EXTRACTO ETANÓLI-}

CO: Se identificaron las características organolépticas del extracto, y se realizó el análisis microbiológico para lo se utilizó placas petrifilm con $1 \mathrm{~mL}$ del extracto etanólico (20, 40 y $80 \%$ ), incubados a $30 \pm 1{ }^{\circ} \mathrm{C}$ durante $72 \pm 2$ horas.

\section{ENSAYOS CROMATOGRÁFICOS PARA FLAVONOI-}

DES: Se realizó en placas de silica gel $(2 \mathrm{~cm}$ x $10 \mathrm{~cm})$, el sistema de solventes utilizado fue cloroformo - acetona - acido fórmico (7.5:1.7:0.8), y revelado con sulfato de cerio (8).

CONTENIDO DE FLAVONOIDES: Se realizó cuantificación espectrofotométrica de flavonoides totales expresado 
en porcentajes de rutina, según el método de Shinet al con ligeras modificaciones. Se tomó $250 \mu \mathrm{L}$ de extracto con $400 \mu \mathrm{L}$ de agua destilada, se adicionó $38 \mu \mathrm{L}$ de $\mathrm{AlCl}_{3}$ al $10 \% \mathrm{p} / \mathrm{v}$, se agitó y dejó a $25^{\circ} \mathrm{C}$ durante $6 \mathrm{~min}$; se agregó $250 \mu \mathrm{L}$ de $\mathrm{NaOH}$ $1 \mathrm{M}$ y completó el volumen final de 1250 $\mu \mathrm{L}$ con agua destilada. La absorbancia de la reacción fue medida a 400nm en espectrofotómetro UV visible Hewlett Packard. La concentración de flavonoides fue establecida empleando una curva de calibración con un estándar de rutina a diferentes diluciones. Los resultados se expresaron como $\mu$ g de rutina/g de muestra (9) (10).

CONTENIDO DE COMPUESTOS FENÓLICOS: Se realizó cuantificación espectrofotométrica mediante el micrométodo de Folin- Ciocalteau. Una alícuota $10 \mu \mathrm{L}$ de extracto se aforó a $100 \mathrm{~mL}$. A 10 $\mathrm{mL}$ de la dilución se añadieron $1.5 \mu \mathrm{L}$ del reactivo de Folin-Ciocalteau (ácido fosfotúngtico con ácido fosfomolibdico) en la oscuridad, se adicionó $2 \mu \mathrm{L}$ de carbonato de sodio al $20 \%$. Se midió la absorbancia a $700 \mathrm{~nm}$ (11).

La concentración de compuestos fenólicos totales se determinó empleando una curva de calibración con ácido gálico como estándar. Fue expresado en $\mu \mathrm{g}$ de ácido gáli$\mathrm{co} / \mathrm{mL}$ de extracto (12) (13).

DETERMINACIÓN DE TANINOS: Se realizó determinación colorimétrica de taninos expresados como ácido tánico por el método de Folin- Ciocalteau. A $0.1 \mu \mathrm{L}$ de extracto etanólico se aforó a $100 \mathrm{~mL}$ con agua destilada. A $10 \mathrm{~mL}$ de la dilución se añadió $2 \mu \mathrm{L}$ de reactivo de Folin - Ciocalteau, protegido de la luz para evitar la oxidación. La concentración de taninos se determinó utilizando ácido tánico a diferentes diluciones. Los resultados se expresaron como $\mu \mathrm{g}$ de ácido tánico/gramo de tejido vegetal (14).

EVALUACIÓN DE LA ACTIVIDAD CICATRIZANTE: Se utilizó 18 ratones albi- nos (Mus musculus) distribuidos al azar en 6 grupos de 3 ratones, con un peso entre $35 \mathrm{y} 40 \mathrm{~g}$, de 2 meses de edad provenientes del bioterio de la ESPOCH, a condiciones ambientales: humedad relativa de $50 \pm 10 \%$, temperatura de $22 \pm$ $2{ }^{\circ} \mathrm{C}$, con periodos de fotoluminiscencia de 12 horas de luz y 12 horas de oscuridad. Se alimentaron con dieta balanceada y agua clorada. El período de ambientación fue durante 15 días. Se conservó normas asépticas durante el periodo del ensayo (15) (16).

Terminado los días de aclimatación se procedió a depilar la mitad del tercio superior del lomo (dorso del animal) de cada ratón, a las 48 horas al no observarse irritación en la piel, se procedió a realizar las heridas de $2 \mathrm{~cm}$ de longitud por $2 \mathrm{~mm}$ de profundidad con la ayuda de un bisturí en condiciones asépticas. Posteriormente se administraron los tratamientos a los grupos experimentales de la siguiente manera (17) (18).

- $\quad \mathrm{CN}$ (control negativo) ratón con herida sin tratamiento.

- $\quad$ CL (control positivo) se aplicó con la ayuda de un hisopo crema dérmica comercial a base de acetato de prednisolona/sulfato de neomicina).

- $\quad$ CE (control positivo) se aplicó eterol (fenol 1g, violeta de genciana $0.4 \mathrm{~g}$, excipiente alcohólico $100 \mathrm{~mL}$ ) (19).

- $\quad$ EC1 se aplicó 3 gotas del extracto al 20\%

- $\quad$ EC2 se aplicó 3 gotas del extracto al $40 \%$

- $\quad$ EC3 se aplicó 3 gotas del extracto al 80\%

Se midió la longitud de la herida por el lapso de 16 días cada 48 horas. Al término del ensayo se procedió a la eutanasia de los animales de experimentación. Se realizó un corte de la piel en el lugar de la herida y fueron conservados en formol para su análisis histopatológico.

El análisis de datos se realizó por el programa IBM SPSS $®$ Statics versión 20.0 aplicando pruebas de estadística descriptiva e inferencial paramétrica ANOVA de un factor y estudios post hoc (test Tukey)

\section{RESULTADOS Y DISCUSIÓN}

En el análisis cualitativo (Tabla 1) se observó en el extracto etanólico la presencia de compuestos con actividad cicatrizante, flavonoides y fenoles. (20) 


\begin{tabular}{|c|c|c|c|c|}
\hline Metabolitos secundarios & Ensayo & $\begin{array}{c}\text { Extracto } \\
\text { etéreo }\end{array}$ & $\begin{array}{c}\text { Extracto etanó- } \\
\text { lico }\end{array}$ & $\begin{array}{c}\text { Extracto } \\
\text { acuoso }\end{array}$ \\
\hline Aceites - grasas & Sudan & + & & \\
\hline Lactonas - cumarinas & Baljet & $+(\downarrow)$ & & \\
\hline Alcaloides & Dragendorf & & $+++(\downarrow)$ & + \\
\hline Alcaloides & Mayer & & + & \\
\hline Alcaloides & Wagner & $(\downarrow)$ & + & \\
\hline Flavonoides & Shinoda & & + & + \\
\hline Azucares reductores & & & & $(\downarrow)$ \\
\hline $\begin{array}{l}\text { Grupo de flavonoides anto- } \\
\text { cianos }\end{array}$ & Antocianos & & + & \\
\hline
\end{tabular}

El extracto etanólico presentó características organolépticas propias de Croton elegans Kunth con un color combinado de verde con café oscuro, de aspecto líquido, olor herbal y sabor dulce por la presencia de azucares.

Los resultados del análisis microbiológico demostraron ausencia de coliformes totales $(10 \mathrm{UFC} / \mathrm{mL})$, moho y levaduras $(<10$ UFC/mL) y aerobios mesófilos (103 UFC/ $\mathrm{mL}$ ) del extracto madre y los extractos al $20 \%, 40 \%$ y $80 \%$, acorde a las especificaciones de la USP 28, el extracto es apto para uso experimental (21).

En el ensayo cromatógrafico según el método de Wagner (1996) se evidenció la presencia de flavonoides como la quercetina (Rf 0.3) y compuestos fenólicos como el ácido isoclorogénico (Rf 0.7), metabolitos relacionados con actividad antinflamatoria y cicatrizante (8)

El contenido total de flavonoides expresado con respecto a la rutina fue en valor de $320 \mu \mathrm{g} / \mathrm{g}$ de muestra.

El contenido de compuestos fenólicos expresados con respecto al ácido gálico fue $2.07 \mu \mathrm{g} / \mathrm{mL}$ de extracto, la concentración de compuestos tánicos totales fue 24.36 $\mu \mathrm{g} / \mathrm{g}$ de muestra, siendo estos los compuestos relacionados con actividad antinflamatoria y cicatrizante (22).

\begin{tabular}{lcc}
\hline Grupo & Cicatrización (días) & Longitud $(\mathbf{c m})$ \\
\hline CN & $15 \pm 0.8$ & $1.16 \pm 0.037$ \\
CL & $13 \pm 0.7$ & $1.12 \pm 0.035$ \\
CE & $11 \pm 0.7$ & $1.13 \pm 0.022$ \\
EC1 & $14 \pm 0.7$ & $1.11 \pm 0.048$ \\
EC2 & $10 \pm 0.5$ & $0.85 \pm 0.010$ \\
EC3 & $8 \pm 0.4$ & $0.91 \pm 0.045$ \\
\hline
\end{tabular}

Tabla 2. Efecto cicatrizante del extracto de Croton elegans kunth.

En el análisis del efecto cicatrizante del extracto de Croton elegans Kunth evaluada mediante la media del tiempo en días para la cicatrización (Tabla 2), el tratamiento EC3 presentó un efecto mayor frente a los otros tratamientos obteniendo un promedio de cicatrizacion de 8 días, se sugiere que la mayor presencia de compuestos fenólicos en el extracto al $80 \%$ consiguió acelerar el proceso. (4)

\begin{tabular}{|c|c|c|c|c|c|c|}
\hline 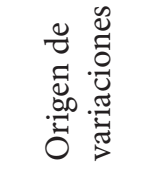 & 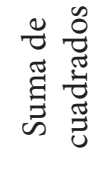 & 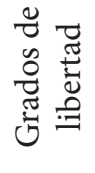 & 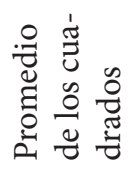 & 䓵 & 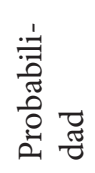 & 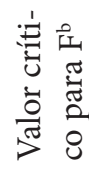 \\
\hline $\begin{array}{l}\mathrm{E} n \mathrm{t} \text { e } \\
\text { grupos }\end{array}$ & 96.94 & 5 & 19.39 & 14.54 & 0.00 & 3.11 \\
\hline $\begin{array}{l}\text { Dentro de } \\
\text { los gru- } \\
\text { pos }\end{array}$ & 16 & 12 & 1.33 & & & \\
\hline Total & 112.94 & 17 & & & & \\
\hline
\end{tabular}

a. Fisher tabulado (Ftab)

b. Fisher calculado (Fcal)

Tabla 3. ANOVA de un factor para los días de cicatrización 
De acuerdo a los datos de ANOVA (Tabla 3) se pudo observar que existe variación entre grupos de acuerdo al valor de Fcal (3.11) en comparacion en Ftab (14.54) lo que indica que el efecto del extractro alcohólico de Croton elegans Kunth en las heridas presenta una variacion significativa al $\mathrm{p}=0.05$ frente a los entes de control.

\section{Dias de cicatrización}

\begin{tabular}{lcccc}
$\begin{array}{l}\text { HSD Tukey } \\
\text { Tratamiento }\end{array}$ & \multicolumn{3}{c}{ Subconjunto para alfa = 5 } \\
\cline { 3 - 5 } & & 1 & 2 & 3 \\
EC3 & 3 & 8.67 & & \\
EC2 & 3 & 10.00 & & \\
CE & 3 & 11.00 & 11.00 & 13.3 \\
CL & 3 & & 13.3 & 14.3 \\
EC1 & 3 & & & 15.00 \\
Cont. Neg. & 3 & & & .518 \\
Sig. & & 2.06 & 2.06 & \\
\end{tabular}

Se vizualiza las medias para los grupos en los subconjuntos homogeneos

a. Utiliza el tamaño de la muestra de la media armónica $=3,00$

Tabla 4. Test de Tukey respecto a los días de cicatrización

Según la prueba de subconjuntos de grupos de Tukey (Tabla 4) con un nivel de significancia 0.05 , se observaron tres subgrupos, de los cuales el tratamiento EC3 (extracto al 80\%) presentó mayor efectividad con 8.67 días en promedio.

En el analisis histopatologico macroscopico de los cortes de las pieles de los animales de experimentación los cinco grupos presentaron coloración blanca y aspecto liso. El grupo EC3 correspondiente al extracto al $80 \%$ la piel esta cubierta de pelaje y no se observa huella de la herida.
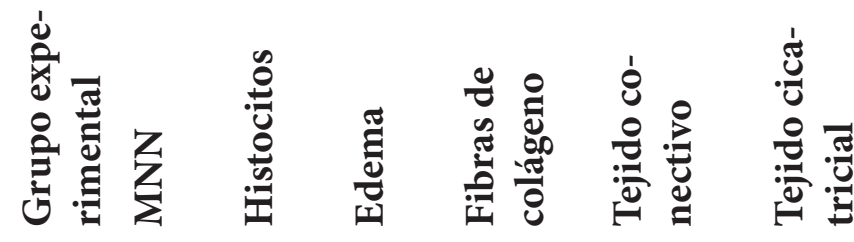

\begin{tabular}{lcccccc}
\hline $\mathrm{EC3}$ & + & - & ++ & ++ & 100 & 90 \\
$\mathrm{EC2}$ & + & - & - & ++ & 80 & 90 \\
$\mathrm{EC} 1$ & + & - & - & ++ & 50 & 80 \\
$\mathrm{CE}$ & + & - & + & ++ & 80 & 100 \\
$\mathrm{CL}$ & + & - & - & ++ & 100 & 80 \\
$\mathrm{CN}$ & + & - & ++ & - & 60 & 100 \\
\hline
\end{tabular}

Tabla 5. Analisis microscopico histopatologico de pieles de ratones
Mediante el análisis microscópico (Tabla 5) de las placas elaborados en base a la piel se observó los tejidos reconstituidos interiormente, siendo favorable el extracto al $80 \%$, pudiendo atribuirse químicamente la actividad cicatrizante a la presencia de compuestos fenólicos de tipo flavonoide porque estos metabolitos intervienen en la cicatrización al evitar la liberación de prostaglandinas e histaminas, evitan la migración de elementos formes (neutrófilos y otros). Además, estabilizan la membrana celular capturando a los radicales libres presentes, evitando así el daño celular y activando el complejo sistema bioquímico para la regeneración del tejido. (20) (23)

\section{CONCLUSIONES}

Mediante el tamizaje fitoquímico se determinó la presencia de metabolitos secundarios de interés farmacológico, siendo los flavonoides identificados en el extracto etanólico importantes para la presente investigación.

El ensayo cromatográfico evidenció la presencia de flavonoides y compuestos fenólicos en el extracto etanólicoe.

El contenido total de flavonoides $(320 \mu \mathrm{g} / \mathrm{g}$ de muestra), compuestos fenólicos $(2.07 \mu \mathrm{g} /$ $\mathrm{mL}$ de extracto) y compuestos tánicos totales $(24.36 \mu \mathrm{g} / \mathrm{g}$ de muestra), lo cual es un indicio de potencial farmacológico relacionados con actividad antinflamatoria y cicatrizante de Croton elegans Kunth.

Mediante el estudio de restauración de heridas provocadas a ratones (Mus musculus), el extracto etanólico de al 80\% de hierba mosquera presentó una diferencia significativa al ser comparados con la crema base de acetato de prednisolona/sulfato de neomicina, el eterol, y el control, corroborándose con el respectivo estudio histológico, en el que se presentó una reconstitución del tejido más rápida. Se evidenció con bases científicas la actividad cicatrizante de Croton elegans Kunth. 
1. L. de la Torre, H. Navarrete, P. Muriel M., M. J. Macía \& H. Balslev (eds.). Enciclopedia de Plantas útiles del Ecuador Quito \& Aarhus: Herbario QCA \& Herbario AAU; 2008.

2. P.M. Jørgensen SLY. Catalogue of the Vascular Plants of Ecuador St. Louis,: Missouri Botanical Garden Press; 1999.

3. León-Yánez S, Valencia R, Pitman N, Endara L, Ulloa C. Libro rojo de las plantas endémicas del Ecuador. 2nd ed. Quito: Publicaciones del Herbario QCA, Pontificia Universidad Católica; 2012.

4. Herrera C, Pérez Y, Morocho V, Armijos C, Malagón O, Brito B, et al. PRELIMINARY PHYTOCHEMICAL STUDY OF THE ECUADORIAN PLANT. J. Chil. Chem. Soc. 2018; 63(No 1).

5. Dominguez X. Métodos de Investigación Fitoquimica. 1st ed. México: Limusa ; 1973.

6. Muñoz J. Guia para el analisis de vegetales Universidad Central del Ecuador, editor. Quito: Universitaria; 1982.

7. Luck O. Investigación Fitoquímica: Métodos de estudios de productos naturales. 3rd ed. Lima: Fondo Editorial Pontifice Universidad Catlica del Perú ; 2016.

8. Bladt S. Plant Drug Analysis. A Thin Layer Chromatography Atlas. 2nd ed. Berli-Alemania: Springer; 1996.

9. Játiva C. Texto básico de farmacognosia de los vegetales a las medicinas Riobamba: Editorial ESPOCH; 2010.

10. Makka H, Bluemel M, Borowy N, Becker K. Gravimetric determination of tannins and their correlations with chemical and protein precipitation methods. J. Sci. Food Agrio. 1993;(61): p. 161-165.

11. García E, Fernández I, Fuentes A. Determinación de polifenoles totales por el método de Folin Ciocalteu. Universitat Politècnica de València. Escuela Técnica Superior de Ingeniería Agronómica y del Medio Natural. 2015 Jun.

12. Ricco R, Wagner M, Portmann E, Reides C, Gurdi A, Carballo M. Analisis de polifenoles, actividad antioxidante y genotoxicidad en especie argentinas de Lippia y Aloysia (Verbenaceae). Boletín Latinoamericano y del Caribe de Plantas Medicinales y aromáticas. 2010; 9(5): p. 388-396.

13. Maksimovic Z, Malencic D, Covacevic N. Polyphenol contents and antioxidant activity of Mayadics stigma extracts. Biores. Tech. 2005; 96: p. 873 - 877.

14. Kasay MI, Humán J, Guerrero M. Estudio cualitativo y cuantitativo de taninos de la OENOTHERA ROSEAL L'HÉR. EX AITON. Rev.Per.Quím.Ing.Quím. 2013; 16(1): p. 13-19.

15. Mancebo B, Sánchez L, Díaz S, Gouchea C, Regalado A, Escobar A, et al. Efecto cocatrizante de la pasta de clorofila-caroteno de Pinus caribaea var. sobre heridas abiertas asépticas. Revista Cubana de Plantas Medicinales. 2011; 16(1): p. 24-33.

16. Victoria M, Morón F. Bioetica en experimentación animal para validar usos de planas medicinales en el Laboratorio Central de Farmacología. Revista Cubana de Plantas Medicinales. 2010; 15(3): p. 157-158.

17. Gónzalez r. Modelos experimentales para la evaluación de la acción cicatrizante de medicamentos. Rev Cubana Farm. 2002; 36(189-196).

18. Gallardo J, Barboza L. Efecto cicatrizante del gel elaborado del látex de Croton lechleri "Sangre de Drago". Revista Cientifica Ciencias Médicas. 2015; 18(1): p. 10 - 16.

19. Manualzz. Veterinario 2015. [Online].; 2015 [cited 201506 15. Available from: https://manualzz.com/ doc/5605682/veterinario-2015.

20. Soriano M, Bonilla P, Arroyo Acevedo J, Pereyra S. Actividad cicatrizante tópica de los metabolitos secundarios en el extracto etanólico de hojas de Senecio culcitoides Weed. Folia dermatol. 2004 dic; 15(3): p. 155-159.

21. Rockville. US. United States Pharmacopeial Convention, Inc. USP 28. The united states pharmacopeia. NF 23. The national formulary; 2005.

22. Sanchez J. Efecto de la quercetina y la rutina frente a daño oxidativo inducido en eritrocitos con distintos contenidos de colesterol. Universidad de Salamanca. Departamento de Bioquímica y Biología Molecular ed. Salamanca: Tesis Doctoral; 2009.

23. Valencia C. Cicatrización: Proceso de reparación tisular. Aproximaciones terapeúticas. Folia dermatol. 2004; 15(3): p. 155-159. 